\title{
Téoros
}

Revue de recherche en tourisme

\section{L’aménagement des Corridors Verts}

\section{Les secrets du praticien}

\section{André Proulx}

Volume 16, numéro 1, printemps 1997

La région touristique de l'Estrie / Cantons-de-l'Est

URI : https://id.erudit.org/iderudit/1074930ar

DOI : https://doi.org/10.7202/1074930ar

Aller au sommaire du numéro

Éditeur(s)

Université du Québec à Montréal

ISSN

0712-8657 (imprimé)

1923-2705 (numérique)

Découvrir la revue

Citer cet article

Proulx, A. (1997). L'aménagement des Corridors Verts : les secrets du praticien.

Téoros, 16(1), 58-61. https://doi.org/10.7202/1074930ar d'utilisation que vous pouvez consulter en ligne.

https://apropos.erudit.org/fr/usagers/politique-dutilisation/ 


\section{L'AMÉNAGEMENT DES CoRRIDORS VERTS}

\section{LES SECRETS DU PRATICIEN}

André Proulx, agronome et directeur général Société de développement des Corridors Verts (Sodécov inc.)

Les corridors verts ne semblent paw, encore aujourd'hui, dégager un sens évocateur dénude de toute ambigülé. Dans les faits, ils ne sont que des sentiers multifonctionnels en milieu naturel où il est permis de pratiquer des activites non motorisées. Plus souvent qu'autrement, les milieux politiques ont tendance à allier les corridors verts uniquenent à une piste cyclable. Cette interprétation singulière est, de toute façon, nécessaire é essentielle pour le dégagenent des crédits pour leur construction. Mais là n'est pas l'essence des propos que je souhaite vous exposer. L'expérience acquise dans mes divers aménagements $m$ 'a vite permis de comprendre et de démontrer aux instances municipales concernées que le projet d'aménagement des corridors verts de la région sherbrookoise, en occurrence le réseau aLes Grandes-Fourchesw, n'était pas une sinécure. Il y a des écéments pratiques de planification préconstruction très importants à considérer et des critères techniques et administratifs à respecter à la lettre pour rendre un projet réalisable, atrayant, et plus encore, à terme. En voici le résumé.

\section{ÉTABLISSEMENT DU CONCEPT: CRITĖRES DE RÉFLEXION}

Avant de tracer le parcours cyclable sur un territoire donné, il est impératif d'identifier ses forces, ses faiblesses et ses contraintes. Larbitraire n"a pas sa place. C"est d'abord et avant tout sur ces éléments fondamentaux que nous bâtirons l'esquisse du premier itinéraire. Dans un premier temps, il est donc crucial de répertorier et de localiser sur une carte les attraits offerts par les patrimoines naturels, culturels et historiques du secteur couvert par le projet. Des rivières à découvrir, des chutes d'eau, des rapides, des paysages pittoresques ou sauvages, des musées, des restaurants et j'en passe, sont au menu. Très souvent, l "ombre d'un parcours apparaît déjà après cet exercice.

\section{LE MILIEU BIOPHYSIQUE}

Vient ensuite l'analyse biophysique du milieu. Les rivières, montagnes et marais, pour n'en nommer que quelques-uns, sont des obstacles majeurs qu'il faut intégrer à l'étude. Les routes provinciales et les autoroutes offrent le même type de contraintes bien souvent difficilement contournable et qui peuvent occasionner de vilaines surprises si laissées pour compte. Une fois tous ces êléments bien identifiés sur une carte il devient plus facile de dégager des axes de parcours sur lesquels il ne reste plus qu'à exercer un certain raffinage.

Cet exercice est également important pour une autre raison. Une région doit miser sur ses atouts, ses attraits el ses forces pour mettre en oeuvre son plan de développement. Il faut que le tracé retenu offre ce défi de la découverte dont nous souhaitons que chaque utilisateur et touriste s'imprègne pour qu'il en ressente la meilleure sensation. Le tracé doit mettre en valeur la région basé sur les éléments les plus forts ci-haut mentionness pour amoindrir les éléments moins reluisants. Non seulement il appréciera sa randonne mais il y reviendra et qui sait, peut-être avec d'autres.

\section{LES SERVICES}

On ne peut omettre d'inclure dans ce tratvail d'analyse les points de services existant sur le territoire. Les besoins aessentiels $*$ des cyclistes sont simples et fondamentaux. Ils ont faim, soif, chaud et ils ont besoin d'aller aux toilettes. Quoi de plus naturel! Une clientèle familiale va rechercher davantage un parcours où tous ces services sont présents et ì une fréquence relativement élevée. En fait, il faut que le produit s'adapte à la clientèle et si ces services sont inexistants ou inaccessibles, il faudrait sérieusement songer à les intégrer au projet.

Cette attention ne fera qu'augmenter le caractère sécuritaire du produit en plus de réduire les cas de harcèlement et de transgression de la propriété privée. Il faut que le touriste se sente bien accueilli dans la région. Mais si les droits et la quiétude des citoyens avoisinants la piste sont violés, il ne faudrait pas s'attendre à des miracles quant à l'accueil. Il faut donc que le projet soit bien accepté pat la communauté. 


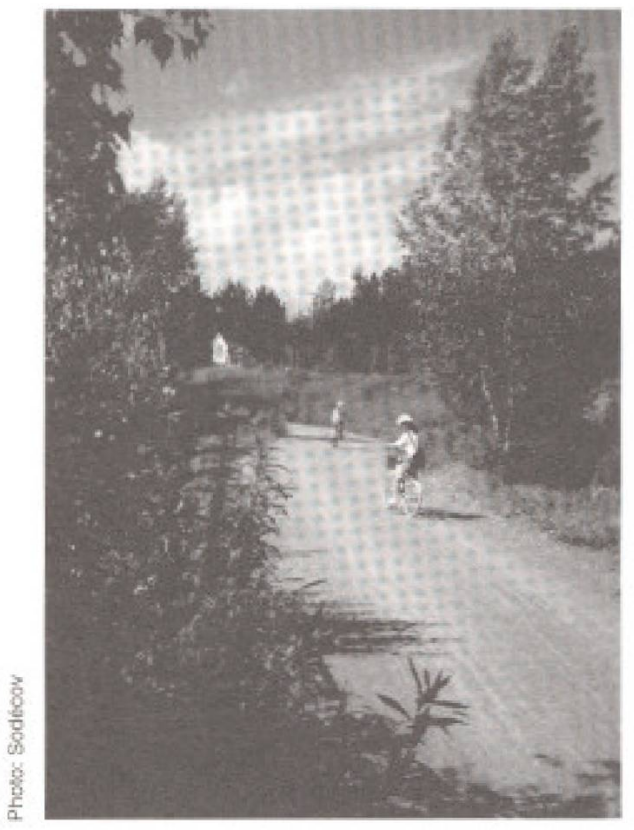

\section{RÉPARTITION DÉMOGRAPHIQUE}

Il est chose courante dans le milieu d'accoler le caractère "récréo-touristique» à tout projet de développement de Corridors verts. Il devient donc important d'intégrer ou d'implanter adéquatement le projet dans le milieu en tenant compte des répartitions démographiques du territoire pour reconnaitre le caractère récréatif du projet. Aussi étonnant que cela puisse paraitre, si le parcours retenu dessert bien les concentrations démographiques, le projet engendrera moins de transports auto pour que le citoyen puisse prendre accès à la piste, réduisant d'autant l'utilisation des aires de stationnement.

\section{LE DESIGN}

La question du design d'un corridor vert en embête plus d'un mais peut être simplifié. Généralement, trois thèmes doivent apparaître sur la feuille de travail; attrayants, diversités, découvertes. Si le parcours choisi rencontre ces objectifs, le projet est assuré de plaire. Pour articuler le développement du design il faut que le concepteur puisse projeter le résultat dans l'avenir, qu'il puisse se l'imaginer. Un dicton dit que anous donnons souvent les cadeaux que nous désirons le plus». Parallèlement, le concepteur ou le maître d'oeuvre devrait se demander: Quel est le design qui me plairait le plus?
Par expérience, nous avons découvert certaines tendances très présentes qui font qu'un parcours plaît plus qu'un autre à un utilisateur donné. Le jeu des contrastes offerts par l'exploitation de la troisième dimension fascine et attire. Le fait d'être tantôt dans une vallée et de voir défiler les collines, et plus tard de se retrouver sur celles-ci et d'y contempler la vallée, prédispose le cycliste à la découverte. De plus, l'exploitation successive des paysages urbains, forestiers, et agricoles, ajoute également à la diversité. Il faut être en mesure tout d'abord de créer l'appétit du découvreur et ensuite de le rassasier graduellement. C'est précisément ce que la Sodécov a appliqué en région. En fait, il faut que le produit puisse maintenir l'intérêt tout au long du parcours. Mais gare à vous si vous ne pouvez combler les attentes de vos utilisateurs.

Finalement, le dernier concept que nous avons mis en oeuvre au niveau du design est celui de la sinuosité du tracé. Nous l'avons appelé le tango du vélo. C'est très sérieux et le citoyen en redemande. Bien aménagé, les méandres, ainsí créés, donnent vraiment la sensation de danser avec le vélo. De plus, ces petits vallons et ces courbes douces, espacés de 150 à 200 mètres, ajouterons à la randonnée une intrigante notion de curiosité et de découverte.

II ne vous reste plus maintenant qu'à colliger, analyser et intégrer tous ces éléments et le tour est joué, ou presque. Vous serez, des lors, en mesure de tracer le parcours préliminaire qui devrait, en principe, inclure les plus beaux attraits de la région. Quelques visites de reconnaissance sur le terrain viendront enfin agrémenter les relevés et valideront la théorie.

\section{LA PLANIFICATION D'AVANT PROJET}

La planification d'avant-projet est l'étape préparatoire avant la mise en oeuvre du chantier. C'est en fait l'étape où l'on consolide la portion étude du tracé par les aspects légaux qui régissent le fonctionnement de notre société. C'est aussi le moment où nous devons nous attarder à l'après-projet, et à une multitude de détails administratifs dont la mise en place est nécessaire pour assurer la pérennité du projet, et de l'investissement.

\section{LES AUTORISATIONS}

Bien que le tracé soit fin prêt sur papier et que tous les partenaires sont convaincus du bien fondé du projet, certaines dispositions additionnelles restent à être prises. En fait, notre sociétét est régit par un certain cadre ríglementaire que le projet doit aussi respecter. Le passage du corridor vert dans une zone riveraine nécessitera, par exemple, I"autorisation préalable du Ministere de l'Environnement et de la Faune (MEF). De même, son passage en zone agricole nécessitera des démarches auprès de la Commission de la protection du territoire agricole du Québec (CPTAQ), et peut-être même une audition. Le Ministère des Transports a aussi son mot à dire si la piste doit emprunter leurs routes ou emprises. Des plans et devis sont habituellement nécessaires dans tous ces cas. Même une municipalité doit ếmettre son avis, et s"assurer que le projet ne contrevient pas à ses propres règlements.

En somme, très tôt dans le projet, il faut inventorier les autorisations nécessaires et s'assurer de leur obtention. Bien qu"en géneral, ces dernières sont émises à l'intérieur d'un délai de quatre à seize semaines, certaines de ces autorisations peuvent prendre jusqu'à douze mois avant leur émission. C'est le cas entre autre pour une demande d'une nouvelle traverse à niveau de voie ferrée.

Dans l'étude du tracé, un promoteur averti aura aussi pris soin de faire l'inventaire et de cartographier les terres, les emprises et les servitudes publiques. Un corridor multifonctionnel est en général beaucoup moins compliqué à aménager sur des terres publiques que sur des propriétés privées. Mais rare sont ces chanceux, Au risque de mettre en péril le projet, il est parfois nécessaire et incontournable d'emprunter des propriétés privées. Tous ces passages doivent, là aussi, être préalablement négociés, autorisés et notariés avec description technique, s'il vous plaît, (donc des frais additionnels). Le terme? Idéalement perpétuel, mais un terme de dix ans est acceptable. Un petit truc en passant; les droits de passage doivent couvrir au minimum le terme de 1'hypothèque si la municipalité va en rè̀glement d'emprunt. C'est important de le savoir avant d'entreprendre les négociations. 


\section{LES PARTENAIRES MUNICIPAUX}

La plupart des projets sont financés en partie ou en totalité par les municipalités partenaires. Ces municipalités adoptent en décembre de l'année précédente le budget de l'année en cour. Bien que cela puisse paraitre simpliste et bien connu, i] faut tout de même que le promoteur en soit informé. Un rappel ne fait jamais de tort

\section{PLANIFICATION BUDGÉTAIRE}

N'ayez crainte, je n'ai pas l'intention d' taler de belles théories sur ce sujet mais simplement vous partager quelques éléments d'ordie général, et par-dessus tout, introduire mon prochain point.

Les possibilités de financement et de partenariat sont tellement variées qu'il est pratiquement impossible de cemer un seul scénario qui puisse servir de modèle. Le projet peut être intermunicipal et appeler plusieurs municipalités à être des partenaires financiers à de multiples niveaux. De même, les gouvernements du Québec et du Canada peuvent aussi devenir partenaires par l'application de différents programmes. Peu importe, ce dont il faut $\mathrm{s}^{\mathrm{a}}$ assurer dans le partage des coûts, c'est l'équité. Il faut que chaque partenaire municipal se sente respecté dans sá capacité de payer et que le bien collectif (le corridor) passe devant les intérêts locaux. Mais attention aux mauvaises surprises. Avons-nous wraiment les moyens de nos ambitions? Il ne faut pas se le cacher, nous sommes pratiquement tous subventionnés pour construire nos corridors verts. Mais avons-nous vraiment les moyens de les entretenir sadéquatements par la suite pour assurer leurs pérennités et la sécurité des utilisateurs. Il ne faut pas attendre à la fin de la période de construction pour le découvrir.

Il importe donc, dès le depart, de bien distinguer, indépendamment et clairement, les postes construction et gestion et d'esquisser des prévisions à l'aide de proformas budgétaires. Il y a rarement des controverses au niveau du partage des coûts de construction. car c'est habituellement la formule *Chacun pour sois qui s'applique. Le consensus n'est pas aussi simple en ce qui a trait au partage des coûts d'entretien d'un projet

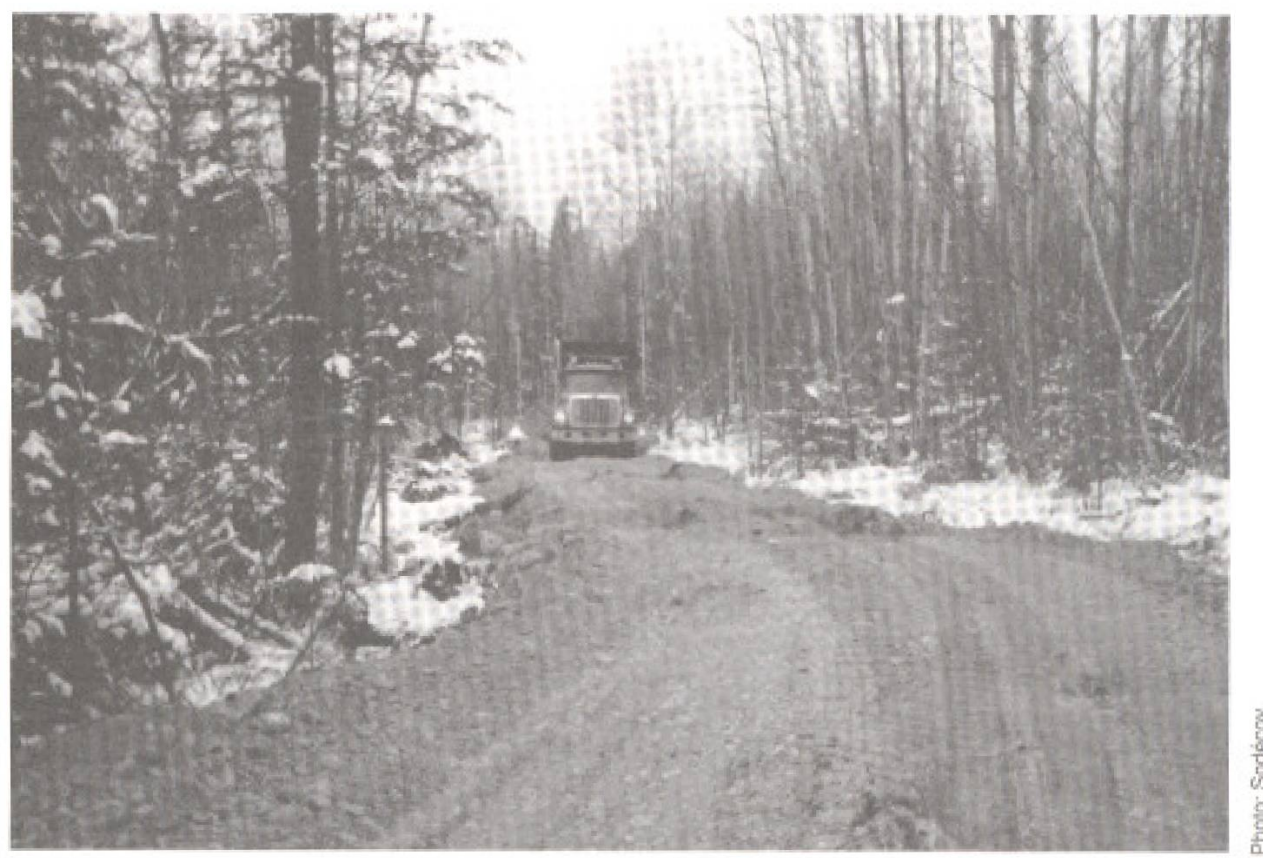

réputé régional. La formule d'equité est plus difficile à trouver et à appliquer.

L'utilisateur d'origine urbaine est beaucoup plus présent sur les sentiers que l'utilisateur rural. Les municipalités rurales peuvent parfois se retrouver avec plusieurs kilomètres de piste sur leur territoire, lesquels seront principalement utilisés par leurs voisins urbains. Il n'y a rien de mal en soi dans ce fait car c'est aussi par souci d'offrir une meilleure qualité de vie à tous les citoyens que ces projets sont réalisés. Cette récente notion de partage force nos philosophies de gestion, «Chacun pour soi $\%$ et Tous pour uns, à s'affronter pour trouver un terrain d'entente, un pacte régional. On $n^{*} y$ echappe pas et mieux vaut l'anticiper avant la phase de construction.

\section{MAITTRISE D'OEUVRE ET STRUCTURE DE GESTION}

Beaucoup d'écrits ont été publiés sur ce sujet. Il n'y a pas de recette miracle et encore moins de modèlé unique applicable à toutes les régions. Ces choix se font en fonction des forces vives du milieu et des opportunités du marché. Dans notre cas, les autorités régionales ont créé un organisme sans but lucratif (OSBL) à qui elles ont confié le mandat d'aménagement. Cette façon de faire permet d'alléger le processus de mise en chantier et de créer un momentum régional autour du projet. Chacune des municipalités concernées siège au conseil d'administration, ce qui leur permet de bien contrôler le projet. En contre partie, chaque municipalité embauche, par convention. l'organisme pour la construction. C'est très efficace, fonctionnel et rentable.

Mais qu'en est-il de la structure de gestion? Dans un contexte de mondialisation où nous devons requestionner l'ensemble de nos façons de faire pour être plus compétitif et rentable, I'OSBL doit aussi se moderniser et $\mathrm{s}^{*}$ ajuster aux nouvelles rétalités. II n'y pas si longtemps, les municipalités créaient des OSBL epara-municipauxs pour bénéficier le plus possible des programmes de subvention pour le développement de la communauté. C'était vrai et cela fonctionnait. Mais aujourd'hui les programmes se raréfient et sont moins alléchants, laissant ces mêmes municipalités avec d'innombrables structures administratives en perte d'autonomie financière. Sans juger de leur pertinence, cela coûte très cher au trésor public. Il y sûrement lieu de simplifier les choses et c'est l'exercice que nous entreprenons actuellement, c'est-àdire: de la réingénierie. Seul l'avenir nous dira si notre vision est la bonne.

Peu importe le type de structure de gestion qui est en place, il $\mathrm{y}$ a tout de mểme un partage des coûts d'entretien et de gestion qui doit se faire, Que ce soit via la MRC par sa formule de répartition de 
la quote-part ou, comme c'est le cas chez nous, la corporation réparti les frais d'entretien entre les municipalites au prorata de la valeur foncière uniformisée, du nombre de kilomètres de piste et de la population. Confiné dans une formule proportionnée selon la réalité, ces éléments constituent le dénominateur commun à une bonne entente à condition qu'ils soient présentés préalablement à la phase construction.

\section{LA CONSTRUCTION}

Vélo Québec, dans son livre wes Sentiers du 2 lième siecles, fait une excellente présentation des techniques d'aménagement. Ce que jaimerais toutefois vous faire partager, ce sont les petits trucs plus englobant que nous avons tous tendance à oublier naturellement.

Tout d'abord, disons que l'aspect de la seccurité me précocupe beaucoup. Nous avons donc développé un réseau avec de multiples accès. Les camions d"entretien et les ambulances doivent pouvoir se rendre rapidement sur le site, sans se retrouver dans un tunnel où la seule sortie possible est à l'autre extrémité. La mểne chose s"applique lors de la construction. Que ce soit sur une ancienne voie ferrée ou en pleine nature, le camion de dix roues doit faire marche arrière pour décharger sa cargaison. Et pas plus d"un camion à la fois peut rouler dans l'étroit corridor. Sur de grandes distances, il y a une énorme perte de temps et d'efficacité. Nous avons atténué cette difficulté en pratiquant des aires de virée à environ tous les 500 mètres qui, maintenant, sont devenues des aires de repos. Joindre l'utile à l'agréable.

L’autre élément sur lequel nous nous sommes attardé et pour lequel nous avons investi plusieurs dollars, ce sont les ponts. L'aménagement de ponceaux n'est en soi rien de sorcier et ils sont conçus pour résister à de fortes charges. Mais qu'en est-il des ponts? Existe-t'il des normes pour les pistes cyclables? La réponse fût fort simple. Pour des raisons de sécurité et d'accessibilité en cas d’accident. tous les ponts ont ếté conçus pour supporter les véhicules d'urgence. L'ingénieur est le professionnel qui peut vous traduire cela en pratique. C'est plus dispendieux mais nous croyons qu'il ne faut pas improviser lorsqu'il s"agit de la vie

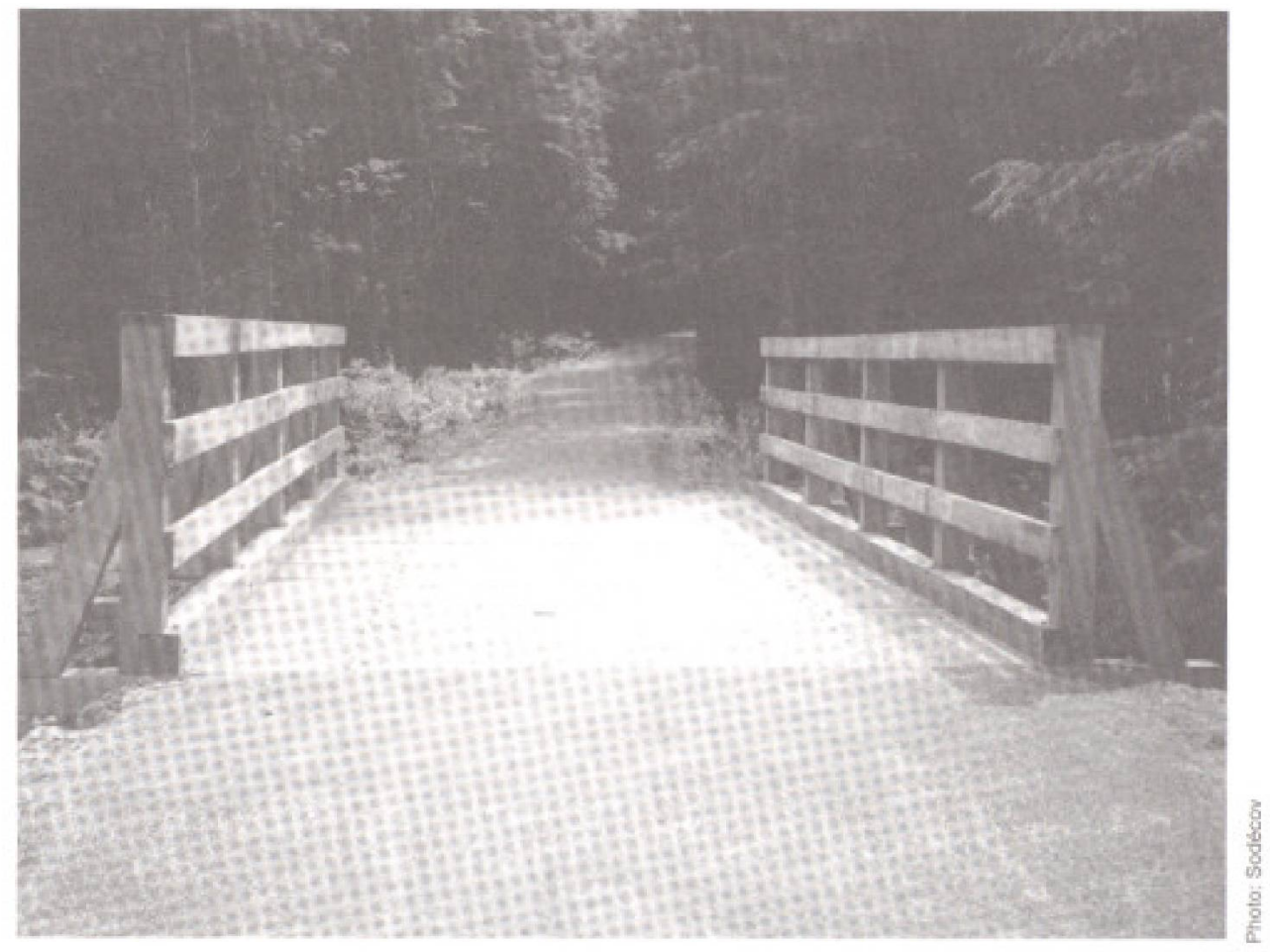

des gens. Et les compagnies d'assurance apprecient beaucoup.

Finalement, qu'elle est la meilleure saison pour construire? La localisation d'une piste en milieu naturel se fait plus facilement l'automne. Les feuilles sont tombées et les plantes herbacées sont écrasées. Le profil du terrain se distingue mieux et $1^{+}$'evaluation des besoins de drainage s"estime avec plus d'aisance. Dans la suite logique des évènements, vient le buchage. $L$ hiver est le temps le plus propice. Pour des raisons environ= nementales et d'économie, les souches sont laissées en terre sauf là où il faut reprofiler le terrain. Le couvert végétal est laissé dans sa forme la plus intacte car il jouera un rôle structurant, comme une géomembrane. En milieu naturel, en forêt, je vous dirais qu'avec l'expérience que nous avons vécu, l'hiver se prête très bien â la construction de piste et c'est en genteral moins dispendieux. Pourquoi? Premièrement, le sol est gelé et la piste requiert moins de matériaux pour la fondation qui supporte les équipements lourds. Deuxièmement, la disponibilité des entrepreneurs nous permet de négocier leur tarif avantageusement. Il est aussi beaucoup plus facile et moins coûteux de traverser les secteurs plus humides. En été, les branches déposées en matelas, ou une géogrille, nous aideront à traverser les secteurs les plus mous. Mais sur une ancienne voie ferrée, l'été est la meilleure pếriode.

Une fois la piste complétée, l"installation de bornes kilométriques et une signalisation directionnelle bien développée viendront coiffer l'aspect sécurité de la piste, l'élément qui met le plus en confiance l'utilisateur touristique.

En résumé, j'ai tenté de décrire brièvement quelques informations pratiques que j’aurais ámé connaître avant d'entreprendre le mandat très édifiant et valorisant du réseau multifonctionnel aLes Grandes-Fourchesw.

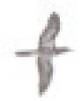

